\title{
Palinotaxonomia de espécies brasileiras de Cheiloclinium Miers (Hippocrateaceae Juss.) ${ }^{1}$
}

\author{
Vania Gonçalves-Esteves ${ }^{2,4}$ e Therezinha Sant'Anna Melhem ${ }^{3}$
}

Recebido em 09/05/2003. Aceito em 17/01/2004

\begin{abstract}
RESUMO - (Palinotaxonomia de espécies brasileiras de Cheiloclinium Miers (Hippocrateaceae Juss.)). O estudo palinotaxonômico de Cheiloclinium Miers teve a finalidade de melhor caracterizar o gênero representado, na América Latina, por 20 espécies. Foram examinados os grãos de pólen de 38 espécimens pertencentes a 14 espécies brasileiras de Cheiloclinium. Os grãos de pólen foram acetolisados, medidos, descritos e ilustrados sob microscopia de luz e eletrônica de varredura. As medidas receberam tratamento estatístico. As principais características polínicas encontradas definem os grãos de pólen como sendo pequenos (médios apenas em $C$. pedunculatum A.C. Sm.), isopolares, suboblatos, oblato-esferoidais ou subprolatos, âmbito subtriangular ou triangular, área polar pequena ou muito pequena (em C. pedunculatum e C. podostemmum (Sandw.) A.C. Sm.), 3-colporados, com endoaberturas lalongadas (circulares somente em C. diffusiflorum (Miers) A.C. Sm.), microrreticulados, reticulados ou granulados. Cheiloclinium é um gênero estenopolínico. Com base nos resultados obtidos em microscopia de luz, no entanto, foi possível a separação de espécies cuja morfologia polínica poderá, efetivamente, contribuir nos estudos taxonômicos deste gênero. Os grãos de pólen de Cheiloclinium não tinham sido analisados previamente.
\end{abstract}

Palavras-chave: Palinologia, Cheiloclinium, Hippocrateaceae

ABSTRACT - (Palynotaxonomy of the Brazilian species of Cheiloclinium Miers (Hippocrateaceae Juss.)). The palynotaxonomic study of Cheiloclinium Miers was conducted in order to contribute to a better characterization of this genus, represented in LatinAmerica by 20 species. Pollen grains from 38 specimens representing 14 Brazilian species were examined. The pollen material was acetolyzed, measured, described and illustrated using ligth and scanning electron microscopy (SEM). The data obtained were statistically analysed. The pollen grains of Cheiloclinium are small in size (medium size only in C. pedunculatum A.C. Sm.), isopolar, suboblate, oblate-spheroidal or subprolate, amb subtriangular or triangular, polar area small or very small (only in C. pedunculatum and C. podostemmum (Sandw.) A.C. Sm.), 3-colporate with lalongate endoaperture (circular only in C. diffusiflorum (Miers) A.C. Sm.) and microreticulate, reticulate or granulate. Cheiloclinium is a stenopalynous genus. However it was possible to elaborate a pollen type key based upon light microscope observations and to distinguish between Cheiloclinium species or species group showing that the pollen characteristics are useful for the taxonomic study of this genus. Pollen grains of Cheiloclinium were never analized before.

Key words: Pollen morphology, Cheiloclinium, Hippocrateaceae

\section{Introdução}

Cheiloclinium Miers, um dos 12 gêneros da família Hippocrateaceae (sensu Smith 1940), é endêmico da América Latina e foi dividido por este autor, em quatro grupos taxonômicos com base nas variações das características vegetativas e reprodutivas (hábito, inflorescência, forma dos estigmas e dos estames); o grupo Cognata é composto por três espécies: $C$. cognatum (Miers) A.C. Sm., C. lineolatum (A.C. Sm.) A.C. Sm. e C. neglectum A.C. Sm. ${ }^{+}$; o grupo Serrata é composto por 10 espécies: C. articulatum (A.C. Sm.) A.C. Sm., C. belizense (Standl.) A.C. Sm., C. diffusiflorum (Miers) A.C. Sm., C. jenmani A.C. Sm. ${ }^{+}$, C. lucidum A.C. Sm. ${ }^{+}$,
C. minutiflorum (A.C. Sm.) A.C. Sm. ${ }^{+}$, C. parviflorum (Miers) A.C. Sm., C. pedunculatum A.C. Sm., C. podostemmum (Sandw.) A.C. Sm. e C. serratum (Camb.) A.C. Sm.; o grupo Hippocrateoides, com cinco espécies, C. gleasonianum (A.C. Sm.) A.C. Sm. ${ }^{+}$, C. hippocrateoides (Peyr.) A.C. Sm., C. klugii A.C. Sm., C. krukovii (A.C. Sm.) A.C. Sm. e C. obtusum A.C. Sm. e, finalmente, o grupo Anomala, formado por duas espécies, C. anomalum Miers e C. glaziovii A.C. Sm. ${ }^{+}{ }^{+}=$espécimen sem pólen $)$.

Smith (1940), ao estudar as Hippocrateaceae da América do Sul, realizou uma revisão taxonômica para o conjunto de espécies brasileiras e sugeriu que os botânicos, nos seus estudos taxonômicos, ficassem

\footnotetext{
Parte da Tese de Doutorado da primeira Autora

2 Museu Nacional, Departamento de Botânica, Universidade Federal do Rio de Janeiro, Quinta da Boa Vista, São Cristóvão, CEP 20940-040 Rio de Janeiro, Brasil, Bolsista de Pesquisa CNPq

3 Instituto de Botânica, C. Postal 4005, CEP 01061-970, São Paulo, Brasil

4 Autor para correspondência: esteves@plugue.com.br
} 
atentos à anatomia do lenho, anatomia floral e morfologia polínica.

$\mathrm{O}$ presente trabalho visa à caracterização morfopolínica do gênero Cheiloclinium, bem como contribuir para melhor circunscrevê-lo, dando sequência aos estudos palinotaxonômicos já realizados com espécies brasileiras dos gêneros Tontelea Aubl. (Gonçalves-Esteves \& Melhem 1998), Salacia L. (Gonçalves-Esteves \& Melhem 2000) e Peritassa Miers (Gonçalves-Esteves \& Melhem 2002), que mostraram a importância do pólen na caracterização das Hippocrateaceae.

\section{Material e métodos}

O material utilizado consta de flores ou botões florais retirados de exsicatas depositadas em herbários nacionais e estrangeiros. No presente estudo, o material foi proveniente dos seguintes herbários: CEPEC, HBR, IAN, INPA, MG, MBM, MO, NY, R, RB, SPF, US. As siglas utilizadas seguem o Index Herbariorum (Holmgren et al. 1990). Os seguintes espécimens foram examinados $\left(^{*}=\right.$ material considerado "padrão"):

Cheiloclinium anomalum - BRASIL. Amazonas: *Humayatem, near Livramento, 12/X-6/XI/1934, B.A. Krukoff 6652 (NY); Pará: Park Indigena do Tumucumaque, Rio Parú do Oeste, Mimão Tirigo, 20/II//1970, P. Cavalcante s.n. (NY2462). C. articulatum - BRASIL. Amazonas: *São Paulo de Olivença, basin of creek Belém, basin of Rio Solimões, 26/X-XI/1936, B.A. Krukoff's 8836 (US). C. belizense - BRASIL. Mato Grosso: Sinop, 20/IX/1985, C.A. Cid Ferreira et al. $6173(\mathrm{NY})$; * Sinop a $142 \mathrm{Km}$ de Peixoto de Azevedo, 5/X/1985, C.A. Cid Ferreira et al. 6354 (NY). C. cognatum - BRASIL. Goiás: próximo à cidade de São Luiz de Montes Belos, 2/III/1978, $H$. Magnago 44 (RB); Maranhão: *Nova Esperança, rio Alto Traiaçu, 16/V/1979, J. Jangoux \& R.P. Bahia 680 (MBM); Colônia Três Satubas, 13/V/1979, J. Jangoux \& R.P. Bahia 5996 (MBM); Mato Grosso: Xavantina, Cachimbo road, 19/XII/1967, A.F. Philcox \& Bertoldo 3594 (RB); Minas Gerais: Santana do Riacho, km 104 ao longo da rod. Belo Horizonte, Conceição do Mato Dentro, 12/I/1981, L. Rossi et al. s.n. (SPF36405); Pará: Almeirim, Estr. da Serraria, s.d., M.R. Santos 675 (HBR19283). C. diffusiflorum BRASIL. Pará: Lago Salgado (Trombetas), 8/IX/1927, A.C. Ducke s.n. (RB20852); Rio Jari, Monte Dourado, 15/X/1968, N.T. Silva 1208 (IAN); *Rio Jari, Monte Dourado, entre Pilão e Repartimento, 28/X/1968, N.T.
Silva s.n. (NY1323). C. hippocrateoides - BRASIL. Amazonas: *Manaus, Cachoeira do Mindú, 22/X/1936, Ducke 317 (NY); Igarapé Curucuhy, São Gabriel, Rio Negro, 8/X/1944, R.L. Fróes 21146 (IAN); Maranhão: rio Turiaçu, Astonas, 6/XII/1978, N.A. Rosa \& H. Vilar 2860 (MG); Pará: Fazenda Santa Rosa, entrance behind town of Itinga do Pará, 26/I/1980, D.C. Daly et al. s.n. (HBR15867); Belém, Catu, 26/VI/1945, A. Ducke 1716 (IAN). C. klugii BRASIL. Pará: *São Félix do Xingu, sub-base Fazenda Dourada, margem direita do rio Dourado, 12/VI/1978, C.S. Rosário 41 (MG). C. krukovii - BRASIL. Amazonas: *São Paulo de Olivença, basin of creek Belém, basin of Rio Solimões, 26/X-XI/1936, B.A. Krukoff's s.n. (MO1261635). C. lineolatum BRASIL. Amazonas: Esperança, Rio Solimões, boca do Javari em direção ao igarapé Jurará, 10/X/1942, A. Ducke 1088 (R); Tefé, 20/X/1948, Murça 1939 (IAN); *Tefé, 20/X/1942, Murça 1309 (US). C. obtusum - BRASIL. Amazonas: *Tefé, Rio Solimões, margem direita do Paraná de Tefé, estr. do Projeto Dendê, km 6, 18/X/1982, C.A. Cid \& J. Lima 3302 (NY); rio Tefé, Muguentaua, 16/VI/1950, R.L. Fróes 26305 (IAN); Igarapé da Chuva, Taraurá, rio Vaupés, entre Ipanoré e confluência com o Rio Negro, 12/XI/1947, R.E. Schultes \& Murça s.n. (IAN52767). C. parviflorum - BRASIL. Amazonas: *Rodovia Manaus-Caracaraí, km 154, BR 174, ManausCaracaraí, 20/IX/1974, G.T. Prance et al. 22725 (MG); Pará: BR 22, vizinhança de Caramema para o Maranhão, próximo da Cachoeira, km 96, 29/X/1965, G.T. Prance \& T.D. Pennington 1793 (IAN). C. pedunculatum - BRASIL. Amazonas: São Paulo de Olivença, basin of creeck Belém, basin of rio Solimões, 26/X-11/XII/1936, B.A. Krukoff's $7^{\text {th }}$ s.n. (MO1176408); *Rio Javari, sete horas acima de Paumari, 16/X/1976, G.T Prance et al s.n. (US2860718). C. podostemmum - BRASIL. Amazonas: *Rio Ituxi, próximo da Boca do Curuquetê, 9/VII/1971, G.T. Prance et al. s.n. (INPA32271). C. serratum - BRASIL. Bahia: Maracás, Gameleiras, 21/XI/1985, G. Hatschbach 50054 \& J.M. Silva (CEPEC); Minas Gerais: Rio Vermelho, Pedra Menina, morro do Ambrósio, 15/VII/1984, R.M. Harley et al. s.n. (SPF33331); Paraná: Arapoti, fazenda do Tigre, 12/III/1960, G. Hatschbach s.n. (MBM34204); Cerro Azul, Sete Quedas, 19/I/1902, R. Kummarov 1686 (MBM); Santa Catarina: *Itajaí, Braço Joaquim Luiz Alves, 16/II/1956, Reitz \& Klein 2716 (HBR); Blumenau, morro Spitzkopf, 6/II/1960, Reitz \& Klein 9528 (HBR). 
Para cada entidade determinou-se um espécime que foi considerado "padrão". Este, sempre que possível, foi selecionado entre os exemplares examinados por Smith (1940). Procurou-se estudar outros espécimes de cada espécie (máximo de cinco), sendo denominados então, "materiais de comparação".

Os grãos de pólen foram acetolisados (Erdtman 1960), medidos até sete dias após a sua preparação (Salgado-Labouriau 1973), foto- e eletromicrografados. Para medidas baseadas em 25 grãos de pólen foram calculados a média aritmética $(\overline{\mathrm{x}})$, o desvio padrão da amostra (s), o desvio padrão da média $\left(\mathrm{s}_{\overline{\mathrm{x}}}\right)$, o coeficiente de variabilidade (CV\%) e o intervalo de confiança a 95\% (Vieira 1981).

Foram realizadas, para cada espécie, somente dez medidas das aberturas (comprimento e largura, excluída a margem) e da espessura das margens das aberturas, bem como das camadas da exina (sexina e nexina). A medida da exina foi feita sempre com os grãos de pólen em vista polar e na região mediana do mesocolpo.

Para a obtenção das eletromicrografias, retirou-se 2 a 3 anteras utilizando-se pinças e estiletes devidamente flambados. Macerou-se as anteras, liberando os grãos de pólen (não acetolisados) sobre suporte previamente recoberto por fita adesiva de carbono dupla face. O material sobre o suporte foi metalizado em ouro por três minutos e o conjunto foi levado para observação ao microscópio eletrônico de varredura (MEV).
A terminologia adotada para a caracterização dos grãos de pólen está de acordo com o glossário de Barth \& Melhem (1988) e Punt et al. (1999).

\section{Resultados}

As espécies de Cheiloclinium estudadas (Fig. 1-50) caracterizam-se por apresentar grãos de pólen de tamanho pequeno na maioria das espécies e médio apenas em C. pedunculatum (Tab. 1 a 3); isopolares; suboblatos (C. anomalum, C. articulatum, C. cognatum, C. krukovii, C. lineolatum), subprolatos (C. hippocrateoides, C. parviflorum) e oblatoesferoidais nas demais espécies. O âmbito é subtriangular ou triangular, são goniotremados, a área polar é pequena na maioria das espécies (Tab. 3) ou muito pequena apenas em $C$. pedunculatum, $C$. podostemmum, 3-colporados, microrreticulados, reticulados, raramente, granulados (Tab. 4).

Os colpos são longos na maioria das espécies ou muito longos em Cheiloclinium pedunculatum e C. podostemmum, estreitos, margem espessa, psilada na maioria das espécies ou reticulada em $C$. diffusiflorum, C. hipocrateoides, C. klugii, C. krukovii, C. lineolatum e $C$. obtusum, endoaberturas circulares em $C$. diffusiflorum e lalongadas nas demais espécies (Tab. 5).

A sexina apresenta-se granulada, sob microscopia de luz, em Cheiloclinium articulatum e C. cognatum. Sob MEV, a sexina do pólen de C. cognatum (Fig. 12) é irregularmente microrreticulada, com áreas psiladas

Tabela 1. Medidas (em $\mu \mathrm{m})$ dos grãos de pólen, em vista equatorial, de espécies de Cheiloclinium Miers ( $\mathrm{n}=25)$.

\begin{tabular}{|c|c|c|c|c|c|c|c|c|c|}
\hline \multirow[b]{2}{*}{ Espécies } & \multicolumn{4}{|c|}{ Diâmetro polar } & \multicolumn{5}{|c|}{ Diâmetro equatorial } \\
\hline & $\begin{array}{l}\text { Faixa de } \\
\text { variação }\end{array}$ & $\overline{\mathrm{x}} \pm \mathrm{s}_{\overline{\mathrm{x}}}$ & $\mathrm{s}$ & $\begin{array}{l}\mathrm{CV} \\
\%\end{array}$ & $\begin{array}{l}\text { Faixa de } \\
\text { variação }\end{array}$ & $\overline{\mathrm{X}} \pm \mathrm{s}_{\overline{\mathrm{x}}}$ & $\mathrm{s}$ & $\begin{array}{l}\mathrm{CV} \\
\%\end{array}$ & $\mathrm{P} / \mathrm{E}$ \\
\hline C. anomalum & $15,5-21,2$ & $18,3 \pm 0,2$ & 0,8 & 4,5 & $20,5-25,9$ & $22,9 \pm 0,2$ & 0,8 & 3,5 & 0,80 \\
\hline C. articulatum & $14,7-17,7$ & $16,1 \pm 0,1$ & 0,5 & 3,0 & $17,2-23,1$ & $20,0 \pm 0,2$ & 0,9 & 4,3 & 0,80 \\
\hline C. belizense & $15,5-19,8$ & $17,7 \pm 0,1$ & 0,7 & 3,7 & $17,9-22,6$ & $20,2 \pm 0,1$ & 0,7 & 3,4 & 0,88 \\
\hline C. cognatum & $14,0-18,4$ & $16,6 \pm 0,2$ & 0,7 & 4,5 & $18,0-21,0$ & $19,5 \pm 0,1$ & 0,5 & 2,5 & 0,85 \\
\hline C. diffusiflorum & $13,6-16,6$ & $15,0 \pm 0,1$ & 0,5 & 3,2 & $14,7-18,2$ & $16,9 \pm 0,1$ & 0,5 & 3,2 & 0,89 \\
\hline C. hippocrateoides & $16,4-20,0$ & $18,8 \pm 0,1$ & 0,6 & 3,0 & $13,8-18,6$ & $16,3 \pm 0,1$ & 0,7 & 4,3 & 1,15 \\
\hline C. klugii & $17,2-22,5$ & $19,6 \pm 0,2$ & 0,8 & 4,0 & $17,6-23,5$ & $20,4 \pm 0,2$ & 0,9 & 4,2 & 0,96 \\
\hline C. krukovii & $14,9-18,8$ & $17,2 \pm 0,1$ & 0,6 & 3,4 & $16,7-22,4$ & $20,2 \pm 0,2$ & 0,8 & 4,2 & 0,85 \\
\hline C. lineolatum & $15,8-20,3$ & $17,5 \pm 0,1$ & 0,7 & 3,9 & $17,0-21,4$ & $20,2 \pm 0,1$ & 0,7 & 3,5 & 0,87 \\
\hline C. obtusum & $17,5-22,5$ & $19,7 \pm 0,2$ & 0,7 & 3,7 & $17,4-23,5$ & $20,6 \pm 0,2$ & 0,9 & 4,3 & 0,96 \\
\hline C. parviflorum & $15,0-20,0$ & $17,4 \pm 0,2$ & 0,8 & 4,4 & $12,5-16,2$ & $14,9 \pm 0,1$ & 0,6 & 3,8 & 1,17 \\
\hline C.pedunculatum & $21,7-25,3$ & $23,4 \pm 0,1$ & 0,6 & 2,5 & $23,5-27,1$ & $25,4 \pm 0,2$ & 0,8 & 3,4 & 0,92 \\
\hline C. podostemmum & $17,8-20,9$ & $19,6 \pm 0,2$ & 0,8 & 4,2 & $17,0-23,5$ & $21,6 \pm 0,2$ & 0,8 & 3,9 & 0,91 \\
\hline C. serratum & $13,7-16,5$ & $15,0 \pm 0,1$ & 0,6 & 3,9 & $14,8-19,7$ & $17,1 \pm 0,2$ & 0,7 & 4,3 & 0,88 \\
\hline
\end{tabular}

$\overline{\mathrm{x}}$ - média aritmética; $\mathrm{s}_{\overline{\mathrm{x}}}$ - desvio padrão da amostra; $\mathrm{s}$ - desvio padrão da média; $\mathrm{CV}$ - coeficiente de variabilidade; P/E = relação entre diâmetro polar e diâmetro equatorial. 
Tabela 2. Valores (em $\mu \mathrm{m}$ ) do intervalo de confiança (I.C.) a $95 \%$ dos diâmetros dos grãos de pólen, em vista equatorial, de espécies de Cheiloclinium Miers ( $\mathrm{n}=25)$.

\begin{tabular}{lcc}
\hline Espécies & $\begin{array}{c}\text { Diâmetro polar } \\
\text { I.C. } 95 \%\end{array}$ & $\begin{array}{c}\text { Diâmetro equatorial } \\
\text { I.C. } 95 \%\end{array}$ \\
\hline C. anomalum & $17,9-18,7$ & $22,5-23,3$ \\
C. articulatum & $15,9-16,3$ & $19,6-20,4$ \\
C. belizense & $17,5-17,9$ & $20,0-20,4$ \\
C. cognatum & $16,2-17,0$ & $19,3-19,7$ \\
C. diffusiflorum & $14,8-15,2$ & $16,7-17,1$ \\
C. hippocrateoides & $18,6-19,0$ & $16,1-16,5$ \\
C. klugii & $19,2-20,0$ & $20,0-20,8$ \\
C. krukovii & $17,0-17,4$ & $19,8-20,6$ \\
C. lineolatum & $17,3-17,7$ & $20,0-20,4$ \\
C. obtusum & $19,3-20,1$ & $20,2-21,0$ \\
C. parviflorum & $17,0-17,8$ & $14,7-15,1$ \\
C. pedunculatum & $23,2-23,6$ & $25,0-25,8$ \\
C. podostemmum & $19,2-20,0$ & $21,2-22,0$ \\
C. serratum & $14,8-15,2$ & $16,7-17,5$ \\
\hline
\end{tabular}

mais proeminentes que se apresentam em microscopia de luz como saliências e grânulos intercalados com perfurações esparsas, semelhantes às observadas no detalhe da Fig. 5 de $C$. articulatum.

Nos grãos de pólen com exina microrreticulada ou reticulada, os muros do retículo apresentam-se lisos ou ondulados devido a columelas proeminentes nos pontos de interseção dos retículos, com lúmens lisos, de tamanho e forma variáveis, podendo apresentar diâmetro menor nos pólos e/ou próximo às aberturas
(Tab. 4), camada infratectal simplescolumelada, mais espessa no mesocolpo e gradativamente mais fina junto às aberturas, sexina mais espessa, menos espessa do que a nexina ou de igual espessura (Tab. 6).

Observações sob MEV (Fig. 19) confirmam a presença de exina microrreticulada e de muros ondulados para os grãos de pólen de Cheiloclinium hippocrateoides.

Os grãos de pólen de Cheiloclinium lineolatum apresentam sexina reticulada com muros largos (Fig. 29-31). Sob MEV, constata-se a presença de lúmens com grânulos esparsos (Fig. 26). Por sua vez, a exina do pólen de C. obtusum, sob MEV, mostra-se microrreticulada (Fig. 35), com muros mais salientes na sua porção mediana dando, sob microscopia de luz, a impressão de que o lúmen é maior, e que a ornamentação seria do tipo sexina finamente reticulada.

Os materiais de comparação examinados confirmam a presença de pólen de tamanho médio apenas em C. pedunculatum (Tab. 7) e mostram que as médias aritméticas das medidas do diâmetro polar dos materiais de comparação situaram-se sempre dentro da faixa de variação do material padrão. Para o diâmetro equatorial, as medidas encontradas mostram uma certa variabilidade mas, frequentemente, também ficam incluídas na faixa de variação do material padrão. Não se conseguiu material polínico de outros espécimes de Cheiloclinium articulatum, C. klugii, C. krukovii e C. podostemmum.

Tabela 3. Medidas (em $\mu \mathrm{m}$ ) dos grãos de pólen, em vista polar, de espécies de Cheiloclinium Miers; lado do apocolpo (LA); índice da área polar (IAP) $*_{\mathrm{n}}=10$.

\begin{tabular}{|c|c|c|c|c|c|c|c|}
\hline \multirow[t]{2}{*}{ Espécies } & \multirow[b]{2}{*}{ Faixa de variação } & \multicolumn{3}{|c|}{ Diâmetro equatorial $(n=25)$} & \multicolumn{3}{|c|}{ LA* } \\
\hline & & $\overline{\mathrm{X}} \pm \mathrm{s}_{\overline{\mathrm{x}}}$ & $\mathrm{s}$ & I.C. $95 \%$ & $\mathrm{CV} \%$ & $\bar{x}$ & $\mathrm{IAP}^{*}$ \\
\hline C. anomalum & $19,0-23,1$ & $21,3 \pm 0,2$ & 1,0 & $20,9-21,7$ & 4,8 & 6,3 & 0,30 \\
\hline C. articulatum & $16,3-19,1$ & $17,6 \pm 0,2$ & 0,7 & $17,2-18,0$ & 4,2 & 4,9 & 0,28 \\
\hline C. belizense & $16,8-22,1$ & $19,4 \pm 0,2$ & 0,8 & $19,0-19,8$ & 4,1 & 4,9 & 0,25 \\
\hline C. cognatum & $17,6-21,8$ & $19,4 \pm 0,1$ & 0,7 & $19,2-19,6$ & 3,4 & 4,8 & 0,25 \\
\hline C. diffusiflorum & $14,4-17,7$ & $16,1 \pm 0,1$ & 0,5 & $15,9-16,3$ & 3,2 & 4,6 & 0,29 \\
\hline C. hippocrateoides & $14,4-19,0$ & $17,0 \pm 0,2$ & 0,7 & $16,6-17,4$ & 4,3 & 4,3 & 0,25 \\
\hline C. klugii & $17,5-22,0$ & $19,2 \pm 0,1$ & 0,7 & $19,8-20,2$ & 3,6 & 5,1 & 0,27 \\
\hline C. krukovii & $17,6-21,8$ & $20,0 \pm 0,1$ & 0,7 & $19,1-19,5$ & 3,4 & 7,0 & 0,35 \\
\hline C. lineolatum & $16,7-21,3$ & $19,3 \pm 0,1$ & 0,7 & $19,0-19,4$ & 3,5 & 7,5 & 0,39 \\
\hline C. obtusum & $17,6-24,1$ & $21,4 \pm 0,2$ & 0,9 & $21,0-21,8$ & 4,4 & 8,3 & 0,39 \\
\hline C. parviflorum & $15,0-18,8$ & $16,3 \pm 0,1$ & 0,6 & $16,1-16,5$ & 3,5 & 6,1 & 0,37 \\
\hline C. pedunculatum & $24,1-26,6$ & $25,0 \pm 0,1$ & 0,7 & $24,8-25,2$ & 2,7 & 6,1 & 0,24 \\
\hline C. podostemmum & $18,9-22,0$ & $20,7 \pm 0,2$ & 0,8 & $20,3-21,1$ & 3,8 & 4,0 & 0,19 \\
\hline C. serratum & $15,7-19,3$ & $17,2 \pm 0,1$ & 0,5 & $17,0-17,4$ & 3,1 & 4,8 & 0,28 \\
\hline
\end{tabular}

$\overline{\mathrm{X}}$ - média aritmética; $\mathrm{s}_{\overline{\mathrm{x}}}$ - desvio padrão da amostra; s - desvio padrão da média; I.C. - intervalo de confiança; CV - coeficiente de variabilidade 

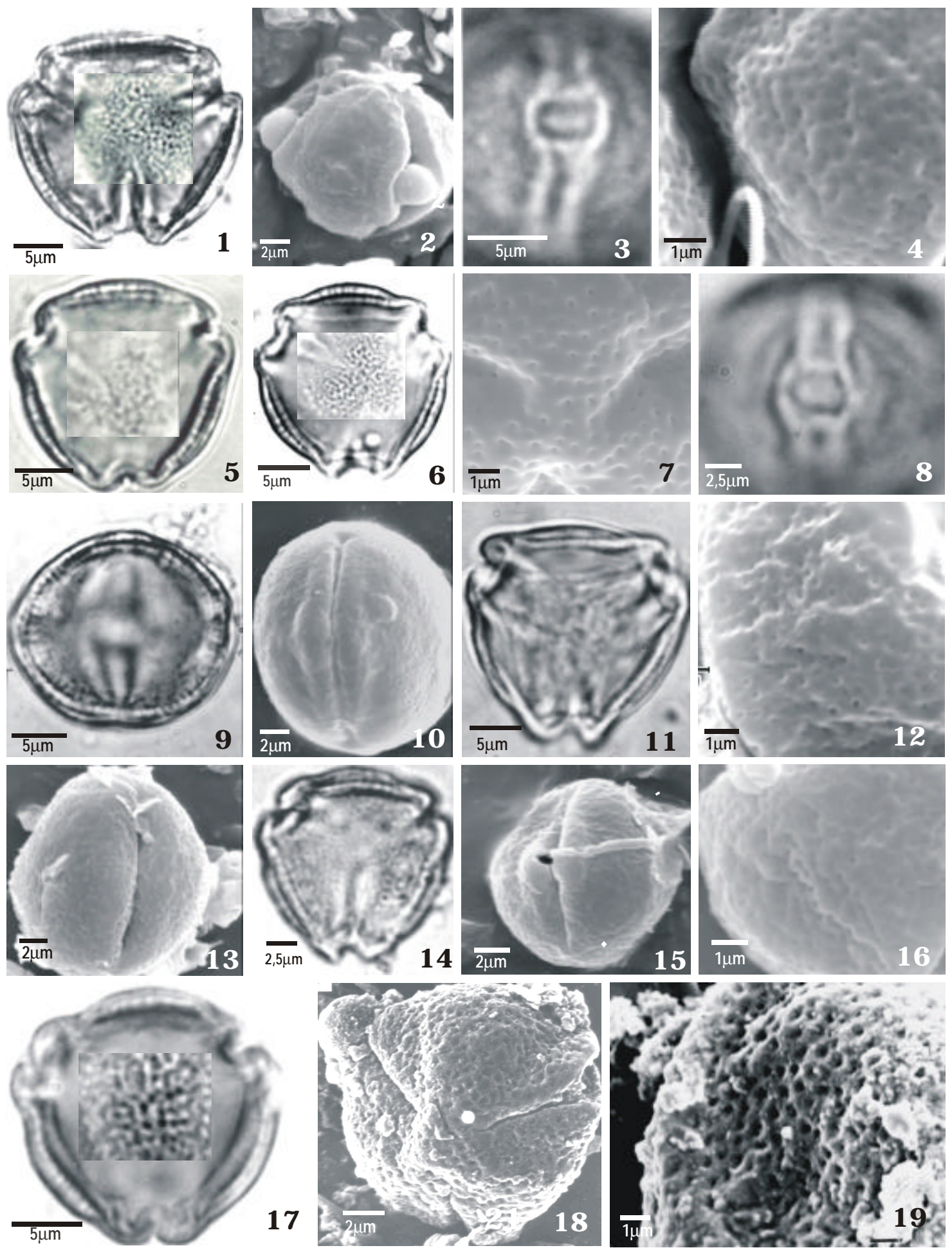

Figuras 1-19. Fotomicrografias e eletromicrografias dos grãos de pólen de espécies de Cheiloclinium Miers. 1-4. C. anomalum - 1. Vista polar em corte óptico e superfície. 2-4. Vista equatorial: 2. Superfície e aberturas. 3. Detalhe da abertura. 4. Superfície. 5. C. articulatum - Vista polar, corte óptico e superfície. 6-10. C. belizense - 6. Vista polar, corte óptico e superfície. 7. Superfície na região do apocolpo. 8. Detalhe da abertura. 9-10. Vista equatorial: 9. Corte óptico. 10. Vista equatorial de um pólen deformado na preparação para MEV. 11-13. C. cognatum - 11. Vista polar, corte óptico. 12. Superfície. 13. Vista equatorial de um pólen deformado na preparação para MEV. 14-16. C. diffusiflorum - 14. Vista polar: corte óptico e superfície. 15. Vista equatorial, abertura. 16. Superfície. 17-19. C. hippocrateoides - 17-18. Vista polar: 17. Corte óptico e superfície. 18. Superfície. 19. Detalhe da superfície. 

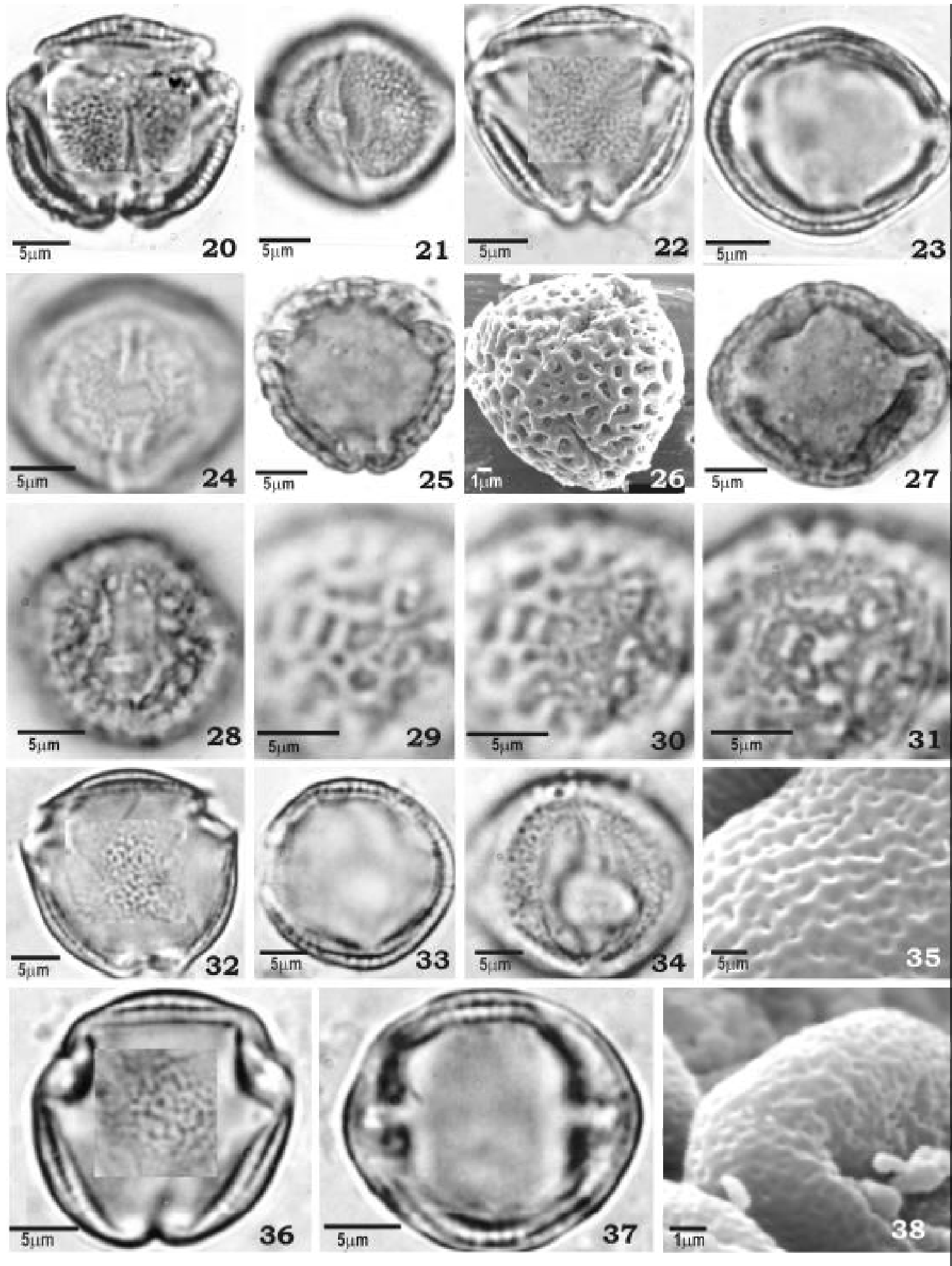

Figuras 20-38. Fotomicrografias e eletromicrografias dos grãos de pólen de espécies de Cheiloclinium Miers. 20-21. C. klugii - 20. Vista polar. corte óptico e superfície. 21. Vista equatorial, abertura. 22-24. C. krukovii - 22. Vista polar, corte óptico e superfície. 23-24. Vista equatorial: 23. Corte óptico. 24. Abertura. 25-31. C. lineolatum - 25-26. Vista polar: 25. Corte óptico. 26. Superfície. 27-28. Vista equatorial: 27. Corte óptico. 28. Abertura. 29-31. Análise de L.O. 32-35. C. obtusum - 32. Vista polar, corte óptico e superfície. $33-34$. Vista equatorial: 33. Corte óptico. 34. Abertura. 35. Detalhe da superfície. 36-38. C. parviflorum - 36. Vista polar, corte óptico e superfície. 37. Vista equatorial, corte óptico. 38. Detalhe da superfície. 

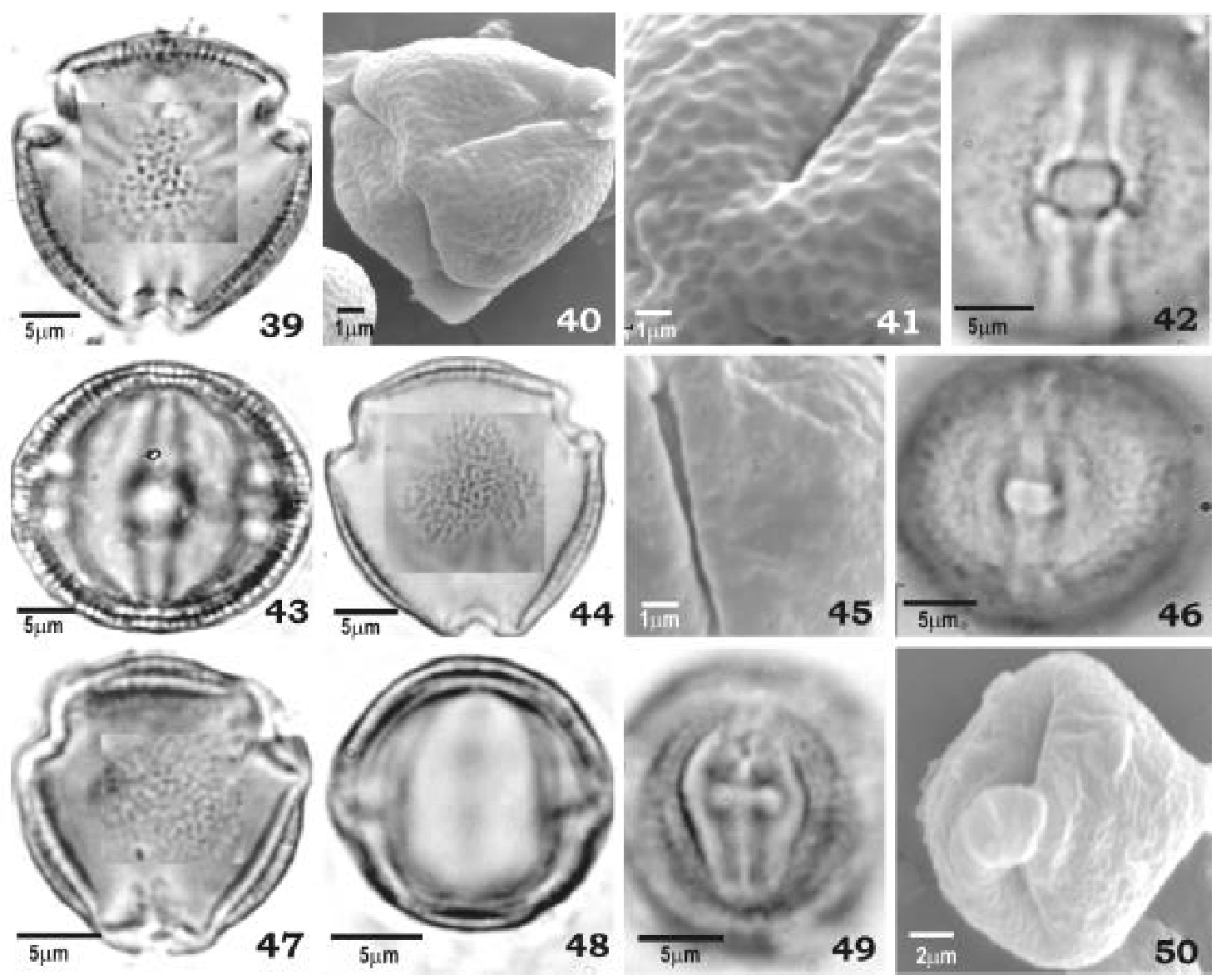

Figuras 39-50. Fotomicrografias e eletromicrografias dos grãos de pólen de espécies de Cheiloclinium Miers. 39-43. C. pedunculatum 39-41. Vista polar: 39. Corte óptico e superfície. 40-41. Superfície. 42-43. Vista equatorial: 42. Abertura. 43. Corte óptico. 44-46. C. podostemmum - 44. Vista polar, corte óptico e superfície. 45-46. Vista equatorial: 45. Detalhe de parte da abertura e superfície. 46. Abertura. 47-50. C. serratum - 47. Vista polar, corte óptico e superfície. 48-50. Vista equatorial: 48. Corte óptico. 49. Abertura. 50. Pólen deformado na preparação para MEV, detalhe da superfície e abertura.

Tabela 4. Características da ornamentação da sexina dos grãos de pólen de espécies de Cheiloclinium Miers, sob microscopia de luz.

\begin{tabular}{|c|c|c|}
\hline Espécies & Figuras & Características da sexina \\
\hline C. anomalum & $1-4$ & microrreticulada, muros ondulados \\
\hline C. articulatum & 5 & granulada, com perfurações esparsas \\
\hline C. belizense & $6-10$ & microrreticulada, muros ondulados \\
\hline C. cognatum & $11-13$ & granulada, com perfurações esparsas \\
\hline C. diffusiflorum & $14-16$ & microrreticulada, muros finamente ondulados \\
\hline C. hippocrateoides & $17-19$ & microrreticulada, muros ondulados \\
\hline C. klugii & $20-21$ & microrreticulada, muros ondulados \\
\hline C. krukovii & $22-24$ & microrreticulada, muros finamente ondulados \\
\hline C. lineolatum & $25-31$ & $\begin{array}{l}\text { reticulada, muros lisos, largos, com perfurações esparsas, columelas consistentes, } \\
\text { nos pólos retículos menores que os do mesocolpo }\end{array}$ \\
\hline C. obtusum & $32-35$ & $\begin{array}{l}\text { reticulada, muros estreitos, finamente ondulados, pólos e mesocolpos com } \\
\text { retículos de igual tamanho }\end{array}$ \\
\hline C. parviflorum & $36-38$ & microrreticulada, muros finamente ondulados \\
\hline C. pedunculatum & $39-43$ & microrreticulada, muros lisos \\
\hline C. podostemmum & $44-46$ & microrreticulada, muros finamente ondulados \\
\hline C. serratum & $47-50$ & microrreticulada, muros lisos \\
\hline
\end{tabular}


Tabela 5. Média (em $\mu \mathrm{m})$ das medidas das aberturas dos grãos de pólen de espécies de Cheiloclinium Miers ( $\mathrm{n}=10)$.

\begin{tabular}{|c|c|c|c|c|c|c|}
\hline \multirow[t]{2}{*}{ Espécies } & \multicolumn{3}{|c|}{ Colpo } & \multicolumn{3}{|c|}{ Endoabertura } \\
\hline & compr. & larg. & margem & compr. & larg. & margem \\
\hline C. anomalum & 13,1 & 0,9 & 1,1 & 2,2 & 3,2 & 1,1 \\
\hline C. articulatum & 9,8 & 0,7 & 0,9 & 1,4 & 1,8 & 1,0 \\
\hline C. belizense & 13,6 & 0,8 & 1,2 & 1,8 & 2,7 & 1,2 \\
\hline C. cognatum & 11,3 & 1,3 & 1,3 & 1,7 & 4,6 & 1,1 \\
\hline C. diffusiflorum & 8,7 & 0,5 & 0,8 & 1,5 & 1,5 & 0,8 \\
\hline C. hippocrateoides & 11,7 & 0,6 & 0,9 & 1,3 & 2,7 & 0,9 \\
\hline C. klugii & 13,7 & 0,5 & 0,9 & 2,1 & 2,5 & 0,8 \\
\hline C. krukovii & 11,7 & 0,6 & 1,0 & 2,1 & 2,8 & 1,0 \\
\hline C. lineolatum & 11,6 & 0,9 & 1,1 & 1,7 & 2,9 & 1,1 \\
\hline C. obtusum & 12,9 & 0,7 & 1,0 & 3,1 & 3,5 & 0,9 \\
\hline C. parviflorum & 10,8 & 0,6 & 0,9 & 1,3 & 2,6 & 0,9 \\
\hline C.pedunculatum & 18,6 & 0,7 & 1,0 & 2,7 & 3,8 & 1,1 \\
\hline C. podostemmum & 13,8 & 0,7 & 1,1 & 2,1 & 2,6 & 1,1 \\
\hline C. serratum & 8,7 & 0,6 & 0,9 & 1,1 & 2,2 & 0,8 \\
\hline
\end{tabular}

Tabela 6. Média (em $\mu \mathrm{m})$ das medidas das camadas da exina dos grãos de pólen de espécies de Cheiloclinium Miers ( $\mathrm{n}=10)$.

\begin{tabular}{lccccc}
\hline Espécies & Exina & Nexina & Nexina 1 & Sexina & Teto \\
\hline C. anomalum & 2,1 & 0,8 & - & 1,3 & 0,8 \\
C. articulatum & 2,0 & 1,0 & 0,7 & 1,1 & 0,4 \\
C. belizense & 1,9 & 0,8 & - & 1,1 & 0,8 \\
C. cognatum & 2,3 & 1,2 & 0,7 & 1,1 & 0,5 \\
C. diffusiflorum & 2,2 & 1,0 & 0,6 & 1,2 & 0,5 \\
C. hippocrateoides & 2,0 & 0,8 & - & 1,2 & 0,7 \\
C. klugii & 2,6 & 1,2 & 0,8 & 1,4 & 0,8 \\
C. krukovii & 2,4 & 1,2 & 0,7 & 1,2 & 0,5 \\
C. lineolatum & 2,3 & 1,0 & - & 1,3 & - \\
C. obtusum & 1,9 & 0,9 & 0,6 & 1,0 & 0,5 \\
C. parviflorum & 2,2 & 1,2 & 0,7 & 1,0 & 0,6 \\
C. pedunculatum & 2,7 & 1,4 & 0,6 & 1,3 & 0,6 \\
C. podostemmum & 1,7 & 0,6 & - & 1,1 & 0,7 \\
C. serratum & 2,2 & 1,2 & 0,7 & 1,0 & 0,5 \\
\hline
\end{tabular}

Tabela 7. Média (em $\mu \mathrm{m})$ dos diâmetros polar (P) e equatorial (E) em vista equatorial e sua relação (P/E) em grãos de pólen de espécimens de comparação de Cheiloclinium Miers ( $\mathrm{n}=10)$.

\begin{tabular}{|c|c|c|c|c|c|c|c|c|c|}
\hline Espécies & $\mathrm{P}$ & $\mathrm{E}$ & $\mathrm{P} / \mathrm{E}$ & Forma & Espécies & $\mathrm{P}$ & $\mathrm{E}$ & $\mathrm{P} / \mathrm{E}$ & Forma \\
\hline C. anomalum & & & & & C. lineolatum & & & & \\
\hline NY2462 & 15,9 & 20,6 & 0,88 & oblato-esferoidal & $\mathrm{R} 75405$ & 20,3 & 23,3 & 0,88 & oblato-esferoida \\
\hline C. belizense & & & & & IAN37083 & 18,0 & 21,0 & 0,86 & suboblata \\
\hline NY6173 & 18,0 & 20,1 & 0,90 & oblato-esferoidal & C. obtusum & & & & \\
\hline C. cognatum & & & & & IAN55080 & 17,5 & 18,1 & 0,97 & oblato-esferoida \\
\hline RB208339 & 18,4 & 19,7 & 0,93 & oblato-esferoidal & IAN52767 & 17,9 & 20,4 & 0,88 & oblato-esferoida \\
\hline MBM100780 & 17,4 & 18,6 & 0,94 & oblato-esferoidal & C. parviflorum & & & & \\
\hline RB156925 & 17,7 & 20,7 & 0,86 & suboblata & IAN117277 & 19,2 & 20,4 & 0,94 & oblato-esferoida \\
\hline SPF36405 & 18,1 & 20,0 & 0,90 & oblato-esferoidal & C. pedunculatum & & & & \\
\hline HBR19283 & 18,0 & 21,0 & 0,86 & suboblata & MO1176408 & 23,3 & 26,8 & 0,87 & suboblatoa \\
\hline C. diffusiflorum & & & & & C. serratum & & & & \\
\hline RB20852 & 16,6 & 17,6 & 0,94 & oblato-esferoidal & CEPEC44517 & 16,5 & 19,7 & 0,84 & suboblatoa \\
\hline IAN127585 & 15,8 & 20,0 & 0,79 & suboblata & SPF33331 & 13,7 & 14,8 & 0,93 & oblato-esferoida \\
\hline C. hippocrateoides & & & & & MBM34204 & 16,5 & 17,5 & 0,95 & oblato-esferoida \\
\hline IAN16583 & 20,0 & 18,6 & 1,08 & prolato-esferoidal & MBM72388 & 16,5 & 17,7 & 0,94 & oblato-esferoida \\
\hline MG66992 & 20,0 & 17,1 & 1,17 & subprolata & HBR23398 & 16,3 & 18,6 & 0,88 & oblato-esferoida \\
\hline HBR15867 & 18,5 & 18,6 & 1,00 & esferoidal & & & & & \\
\hline IAN12502 & 19,9 & 18,4 & 1,08 & prolato-esferoidal & & & & & \\
\hline
\end{tabular}

Chave para as espécies de Cheiloclinium, baseada em microscopia de luz

1. Grãos de pólen médios, intervalo de confiança (I.C.) do diâmetro equatorial entre 25,0 e 25,8 $\mu \mathrm{m}$ ... . pedunculatum

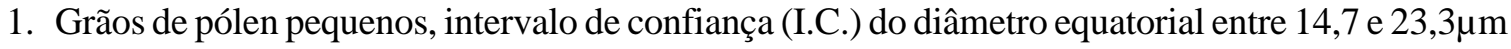

2. Exina granulada

3. Colpo ca. $11,3 \mu \mathrm{m}$ compr., endoabertura nitidamente lalongada ca. $1,7 \times 4,6 \mu \mathrm{m}$ C. cognatum

3. Colpo ca. $9,8 \mu \mathrm{m}$ compr., endoabertura ligeiramente lalongada ca. $1,4 \times 1,8 \mu \mathrm{m}$ C. articulatum

2. Exina reticulada ou microrreticulada 
4. Exina reticulada

5. Área polar com retículos menores que os dos mesocolpos; muros lisos e largos; endoaberturas ca. $1,7 \times 2,9 \mu \mathrm{m}$

C. lineolatum

5. Área polar e mesocolpos com retículos de igual tamanho; muros estreitos, finamente ondulados; endoaberturas ca. $3,1 \times 3,5 \mu \mathrm{m}$

C. obtusum

4. Exina microrreticulada

6. Área polar muito pequena

C. podostemmum

6. Área polar pequena

7. Endoabertura circular ca. 1,5um diâm.

C. diffusiflorum

7. Endoabertura lalongada

8. Nitidamente lalongada, largura o dobro do comprimento

C. hippocrateoides,

8. Ligeiramente lalongada, largura menos do que o dobro do comprimento C. anomalum,

C. belizense, C. klugii e C. krukovii

\section{Discussão}

Não há dados polínicos na literatura para o gênero Cheiloclinium, que pode ser caracterizado, com base nos resultados aqui obtidos, pela presença predominante de grãos de pólen pequenos, área polar pequena, endoabertura lalongada e exina microrreticulada. Variações do tamanho dos grãos de pólen, das áreas polares, da ornamentação da exina e das dimensões das endoaberturas permitiram a separação de espécies ou de grupos de espécies estudadas de Cheiloclinium.

Os grãos de pólen de Cheiloclinium hippocrateoides, $C$. parviflorum e $C$. serratum são similares aos de C. anomalum, C. belizense, $C$. klugii e C. krukovii e a separação desses dois grupos de táxons só foi possível com base nas variações do tamanho e da forma mais ou menos lalongada das endoaberturas.

Não se observou uma correspondência entre os grupos taxonômicos propostos por Smith (1940) e os conjuntos de espécies com grãos de pólen semelhantes. Constituíram bons exemplos dessa divergência: Cheiloclinium cognatum e C. lineolatum, enquadradas no grupo Cognata, cujos grãos de pólen pequenos, com áreas polares pequenas e endoaberturas lalongadas, diferiram entre si, pela ornamentação da exina. Sob microscopia de luz e MEV (Fig. 13 e 26) C. lineolatum apresentou grãos de pólen com exina tipicamente reticulada, bem diferente das demais espécies do grupo (Fig. 26, 29 a 31), enquanto que em C. cognatum a exina é granulada (Fig. 13).

Os demais grupos taxonômicos de Cheiloclinium (Smith 1940), Anomala, Hippocrateoides e Serrata foram caracterizados pela presença de grãos de pólen pequenos, com áreas polares pequenas, endoaberturas lalongadas e exina microrreticulada, mostrando que a morfologia polínica não dá suporte à caracterização destes grupos. Dentro do grupo Serrata, as espécies C. articulatum (com exina granulada sob microscopia de luz), C. diffusiflorum (com endoaberturas circulares), C. pedunculatum (com grãos de pólen de tamanho médio) e $C$. podostemmum (com áreas polares muito pequenas) têm grãos de pólen distintos daqueles das demais espécies deste grupo. O pólen de C. obtusum (grupo Hippocrateoides), sob microscopia de luz, mostra-se finamente reticulado (Fig. 32, 34), devido aos lúmens que se encontram em depressões, mas, sob MEV, notou-se que a sexina é microrreticulada (Fig. 35), típica das demais espécies do grupo.

Em estudo palinotaxonômico referente a 78 espécies, distribuídas em 12 gêneros de Hippocrateaceae ocorrentes no Brasil, Gonçalves-Esteves (1994) concluiu que baseada em características palinológicas, a delimitação dos gêneros Cheiloclinium e Peritassa, era bastante difícil para aquelas espécies com os grãos de pólen em mônades, exceção feita a P. calypsoides (Camb.) A.C. Sm. cujos grãos de pólen apresentaram-se em tétrades. Por outro lado, entre todas as espécies estudadas por Gonçalves-Esteves (1994), a presença de sexina granulada foi constatada, sob microscopia de luz, apenas em Cheiloclinium articulatum, C. cognatum, Peritassa compta (Miers) A.C. Sm. e P. dulcis (Miers) A.C. Sm., mostrando a afinidade polínica dos dois gêneros. Gonçalves-Esteves \& Melhem (2002), após análises mais detalhadas sob MEV das espécies de Peritassa estudadas, confirmaram a presença de uma sexina granulada apenas nos grãos de pólen de $P$. dulcis e, no presente estudo, verificou-se que, sob MEV, o pólen de 
C. cognatum apresentou exina microrreticulada. Para C. articulatum não há dados sob MEV mas, a ornamentação destes dois táxons definidos como granulados (sob microscopia de luz), é idêntica, confirmando a raridade deste tipo de ornamentação entre as Hippocrateaceae.

\section{Agradecimentos}

Ao Laboratório de Microscopia Eletrônica da Universidade de Brasília e à MSc. Maria de Fátima Lopes (in memoriam) da PUC-Rio, por fornecerem condições de trabalho para a obtenção das eletromicrografias; ao $\mathrm{CNPq}$ pelo apoio às autoras através de Bolsa de Produtividade Científica.

\section{Referências bibliográficas}

Barth, O.M. \& Melhem, T.S. 1988. Glossário ilustrado de palinologia. Ed. UNICAMP, Campinas.

Erdtman, G. 1960. The acetolysis method. A revised description. Svensk Botanisk Tidskrift 54: 561-564.
Gonçalves-Esteves, V. 1994. Estudo palinotaxonômico da família Hippocrateaceae Juss. Tese de Doutorado. Universidade de São Paulo, São Paulo.

Gonçalves-Esteves, V. \& Melhem, T.S. 1998. Palinotaxonomia de espécies brasileiras de Tontelea Aubl. (Hippocrateaceae Juss.). Polibotánica 7: 33-54.

Gonçalves-Esteves, V. \& Melhem, T.S. 2000. Palinotaxonomia de espécies brasileiras de Salacia L. (Hippocrateaceae Juss.). Revista Brasileira de Botânica 23(4): 425-440.

Gonçalves-Esteves, V. \& Melhem, T.S. 2002. Palinotaxonomia de espécies brasileiras de Peritassa Miers. (Hippocrateaceae Juss.). Acta Botanica Brasilica 16(2): 193-205.

Holmgren, P.K.; Holmgren, N.H. \& Bainett, L.G. 1990. Index Herbariorum. New York, NYBG, $8^{\mathrm{a}}$ ed., v. 1.

Punt, W.; Blackmore, S.; Nilsson, S. \& Le Thomas, A. 1999. Glossary of pollen and spore terminology. http:// www.biol.ruu.nl/ palaeo/glossary/glos-int.htm

Salgado-Labouriau, M.L. 1973. Contribuição à palinologia dos cerrados. Academia Brasileira de Ciências, Rio de Janeiro.

Smith, A.C. 1940. The american species of Hippocrateaceae. Brittonia 3: 341-571.

Vieira, S. 1981. Introdução à bioestatística. Ed. Campus Ltda, Rio de Janeiro. 